\title{
Computed tomography imaging characteristics of synchronous gastrointestinal stromal tumors in patients with gastric cancer and correlation with clinicopathological findings
}

\author{
ZHENSHAN SHI $^{1}$ and QIAN ZHUANG ${ }^{2}$ \\ ${ }^{1}$ Department of Radiology, The First Affiliated Hospital of Fujian Medical University; \\ ${ }^{2}$ Department of Pharmacy, Union Hospital of Fujian Medical University, Fuzhou, Fujian 350005, P.R. China
}

Received May 27, 2015; Accepted June 27, 2015

DOI: $10.3892 /$ mco.2015.621

\begin{abstract}
This study was conducted to analyze the computed tomography (CT) imaging and clinicopathological characteristics of synchronous gastrointestinal stromal tumors (GISTs) in patients with gastric cancer. We retrospectively reviewed the CT images in 18 cases of immunohistochemically confirmed synchronous GISTs in patients with primary gastric cancer. Relevant histology and clinical data were also obtained. Multi-slice CT (MSCT) identified suspected synchronous GISTs in 26 patients, of which 18 were finally confirmed. Of the 18 patients, 12 were male and 6 female, with a mean age of 69.2 years. All 18 lesions were discovered as a solitary mass, ranging in size from 1.0 to $6.5 \mathrm{~cm}$ (mean, $2.2 \mathrm{~cm}$ ). The lesions were predominantly located in the gastric fundus and were characterized by an ovoid shape, well-defined margins and isodensity, with slight to moderate gradual enhancement on MSCT. There was no evidence of necrosis, hemorrhage or cystic degeneration, irrespective of the tumor size. Synchronous GISTs exhibited spindle cell morphological characteristics and specific immunohistochemical properties, and were classified as being of low or very low malignant potential. In conclusion, since gastric cancer patients with a synchronous GIST are rare, a solitary, ovoid and well-defined mass with slight to moderate gradual enhancement following contrast agent injection may be suggestive of this diagnosis, although detection of synchronous GISTs does not appear to significantly affect therapy and outcome.
\end{abstract}

\section{Introduction}

Gastrointestinal stromal tumor (GIST), the most common type of non-epithelial tumors, is a term used to describe

Correspondence to: Dr Qian Zhuang, Department of Pharmacy, Union Hospital of Fujian Medical University, 20 Chazong Road, Fuzhou, Fujian 350005, P.R. China

E-mail: shizhenshan0206@163.com

Key words: gastrointestinal stromal tumors, gastric cancer, computed tomography a unique group of mesenchymal neoplasms that typically arise in the muscularis propria of the gastrointestinal tract wall, accounting for $0.1-3 \%$ of all gastrointestinal tumors (1). GISTs may develop synchronously with tumors originating from different cell layers, such as cancer, carcinoid and lymphadenoma (2-4). The synchronous development of a gastric GIST and adenocarcinoma is relatively rare. The aim of this study was to conduct a retrospective review of the computed tomography (CT) imaging characteristics of 18 cases of synchronous GIST and primary gastric cancer and evaluate the clinicopathological characteristics of GISTs.

\section{Patients and methods}

Patients. The database of the First Affiliated Hospital of Fujian Medical University was searched and patients who were treated at our hospital between January, 2006 and April, 2013 were identified. A total of 26 patients with histologically proven synchronous GISTs and gastric cancer were investigated. Of these patients, 8 with GISTs sized $<1.0 \mathrm{~cm}$ were excluded, as the preoperative CT images were difficult to characterize with confidence. Therefore, the study cohort consisted of 18 patients (12 men and 6 women; mean age, 69.2 years; and age range, 47-82 years). An Institutional Review Board (IRB) exemption and a waiver of the requirement for written informed consent were obtained to perform this retrospective study. The patient characteristics are summarized in Table I.

CT imaging and pathological analysis. CT imaging was performed using a Toshiba Aquilion 16-slice CT scanner. A total of 18 patients underwent contrast-enhanced CT within 1 week of the operation. After an overnight fast, oral contrast, $750-1,000 \mathrm{ml}$ water, or $2 \%$ oral diatrizoate meglumine was administered to all the patients $30 \mathrm{~min}$ prior to scanning. The scan range was determined depending on the size of the tumor and the distance from the diaphragmatic dome to the inferior border of the liver. Scanning was performed with 5- or 10-mm slice thickness and interval. The CT parameters were $120 \mathrm{kVp}$ and $350 \mathrm{mAs}$. Iopamidol (80-100 ml, $370 \mathrm{mgI} /$ $\mathrm{ml}$ ) was injected intravenously at a rate of $3 \mathrm{ml} / \mathrm{sec}$ and imaging was performed with a dual-phase technique. The technique was performed with a late arterial (portal inflow) 
phase scan at $32 \mathrm{sec}$ and a hepatic venous phase scan at $102 \mathrm{sec}$. Section widths of $2 \mathrm{~mm}$ and reconstruction intervals of $1.25 \mathrm{~mm}$ were used. Two radiologists retrospectively reviewed all radiological studies, with final interpretations by consensus. Each tumor was assessed for location, density, presence of calcifications, size, margin, cyst formation, hemorrhage, necrosis and presence of a tumor capsule. The adjacent organs, fat planes and stomach wall were assessed for evidence of invasion, which was suspected when there was focal enlargement of the organ or anatomic structure in direct continuity with the tumor mass. The tumors were also assessed for the pattern of attenuation during the administration of the contrast material.

The medical records were subsequently reviewed to determine the clinical presentation, therapy and patient course. The pathology records were also reviewed to determine the histological and immunohistochemical characteristics of the lesions, including CD117, CD34, smooth muscle actin (SMA), S-100, Ki-67, desmin (DES), discovered on GIST-1 (DOG1) and platelet-derived growth factor receptor $\alpha$ (PDGFRA) mutation status, when available.

\section{Results}

Clinical presentation. The demographic characteristics of 18 patients with synchronous GISTs are presented in Table I. The major symptoms at presentation included abdominal pain $(n=10)$, abdominal distention $(n=9)$, sour regurgitation $(n=7)$, melena $(n=7)$, nausea and vomiting $(n=3)$, hematemesis $(n=3)$, weight loss $(n=6)$ or unexplained anemia $(n=2)$. Certain patients presented with $\geq 1$ of these symptoms. Each patient had a preoperative histological diagnosis of gastric adenocarcinoma established by gastroscopy and biopsy. Preoperative gastroscopy revealed a cancerous ulcer $(n=10)$, soft tissue mass $(n=5)$, pyloric stenosis and stiffness of the stomach wall $(n=3)$. Five patients had early and 13 patients advanced gastric cancer. Early gastric cancer is defined as tumor confined to the mucosa or submucosa, independent of regional lymph node (LN) metastases, irrespective of the tumor size.

CT findings. Of the 18 synchronous GISTs, 9 were found in the gastric fundus (50\%), 4 in the body (22\%), 2 in the cardia (11\%), 2 in the antrum $(11 \%)$ and 1 in the pylorus $(6 \%)$ (Table I). The median size \pm standard deviation of the GISTs was $2.2 \pm 1.2 \mathrm{~cm}$ (range, $1.0-6.5 \mathrm{~cm}$ ).

All $18 \mathrm{CT}$ scans revealed a solitary, well-marginated, isodense mass with an ovoid shape that was inseparable from the adjacent stomach; the contrast-enhanced CT scans revealed slight to moderate gradual enhancement of these lesions (Fig. 1A-C). The mean unenhanced value was $36 \mathrm{HU}$ (range, 23-45 HU; n=18) and the mean contrast-enhanced value was $54 \mathrm{HU}$ (range, 30-68 HU) in the late arterial (portal inflow) phase; the calculated mean enhancement was $22 \mathrm{HU}$. The mean contrast-enhanced value was $61 \mathrm{HU}$ (range, 37-77 HU) in the hepatic venous phase; the calculated mean enhancement was 12 HU. Two GISTs displayed punctate calcifications (Figs. 1A and 2A); the other GISTs were invariably homogeneous, irrespective of the tumor size. No evidence of necrosis, cystic degeneration or hemorrhage was observed in any of the tumors.
Table I. Characteristics of patients with GISTs $(n=18)$.

\begin{tabular}{lc}
\hline Characteristics & No. \\
\hline Gender & \\
Female & 6 \\
Male & 12 \\
Age, years & \\
Mean & 69.2 \\
Range & $47-82$ \\
Location of GISTs & \\
Gastric fundus & 9 \\
Body & 3 \\
Cardia & 2 \\
Antrum & 2 \\
Pylorus & 1 \\
Tumor size, cm & \\
Mean & 2.2 \\
Range & $1.0-6.5$ \\
Stage of primary gastric cancer & \\
I or II & 5 \\
III & 13 \\
\end{tabular}

GIST, gastrointestinal stromal tumor.

Histopathology. All the GISTs exhibited a spindle cell pattern, without necrosis or cystic changes on histological examination (Fig. 1D). The histopathological examination revealed mitotic rates ranging from 0 to 5 mitoses $/ 50$ high-power fields (hpf). All 18 tumors were considered of low or very low malignant potential (Table II). On immunohistochemical examination, all the tumors expressed CD34 and the c-kit protein $(\mathrm{CD} 117,+$ to +++$)$, which is a type III tyrosine kinase receptor encoded by the c-kit proto-ongogene. Five synchronous GISTs (28\%) exhibited weak focal SMA positivity, but the remaining $13(72 \%)$ were SMA-negative. No GISTs were S-100-positive; 4 GISTs were DOG1-positive and only 1 GIST exhibited DES positivity. The Ki-67 expression varied from 0.01 to 0.05 in 16 patients. Three of the resected specimens underwent molecular genotyping and two PDGFRA mutations were identified.

\section{Discussion}

The coexistence of gastric cancer and GIST is relatively rare, and GISTs are occasionally detected in the gastric serosa or mucosa during surgery (3,5-7). Gastric adenocarcinoma (Fig. 2B) and GISTs are different neoplasms, originating from different cell layers; an accurate pre- and postoperative diagnosis is important. However, when the GIST is subserosal or submucosal, the gastric mucosa has not yet been invaded and endoscopic biopsies may come back as normal.

In the available literature, no case of coexistence of adenocarcinoma and GIST has been detected preoperatively $(2,3,7)$, and there has been no report of CT imaging characteristics of the simultaneous development of adenocarcinoma and GIST in the stomach. In fact, in our cases, the preoperative 
Table II. Immunohistochemistry and risk stratification of GISTs.

\begin{tabular}{|c|c|c|c|c|c|c|c|c|c|c|c|}
\hline Case & Size $(\mathrm{cm})$ & Mitoses (/50 hpf) & CD117 & CD34 & DOG1 & PDGFRA & SMA & S-100 & Des & $\mathrm{Ki}-67(\%)$ & Risk \\
\hline 1 & 1.8 & 1 & + & + & + & & + & - & - & +1 & Very low \\
\hline 2 & 1.0 & 0 & + & + & & & + & - & & +1 & Very low \\
\hline 3 & 2.7 & $<5$ & + & + & + & & - & - & - & +2 & Very low \\
\hline 4 & 1.2 & 0 & - & + & & & - & - & & - & Low \\
\hline 5 & 3.5 & 0 & +++ & +++ & +++ & & - & - & & $+2-3$ & Very low \\
\hline 6 & 1.2 & 1 & + & + & + & & + & - & & +2 & Very low \\
\hline 7 & 1.9 & 5 & + & + & & & - & - & - & - & Very low \\
\hline 8 & 4.2 & 0 & - & + & & & + & - & + & +2 & Very low \\
\hline 9 & 1.0 & $<5$ & + & + & & - & - & - & - & +2 & Low \\
\hline 10 & 6.5 & $<5$ & + & + & & + & - & & & +1 & Low \\
\hline 11 & 1.9 & $<5$ & + & + & & + & - & - & & +1 & Low \\
\hline 12 & 1.5 & 0 & +++ & +++ & & & + & - & & +1 & Very low \\
\hline 13 & 1.0 & $<5$ & + & + & & & - & - & - & +5 & Low \\
\hline 14 & 1.3 & Rare & + & + & & & - & - & - & +1 & Very low \\
\hline 15 & 2.8 & 0 & + & + & & & - & - & - & +2 & Very low \\
\hline 16 & 1.0 & 0 & + & + & & & - & - & & +1 & Very low \\
\hline 17 & 1.0 & $<5$ & + & + & & & - & - & & +1 & Very low \\
\hline 18 & 4.0 & 3 & + & + & & & - & - & & +2 & Low \\
\hline
\end{tabular}

GIST, gastrointestinal stromal tumor; hpf, high-power field; DOG1, discovered on GIST-1; PDGFRA, platelet-derived growth factor receptor $\alpha$; SMA, smooth muscle actin; Des, desmin.
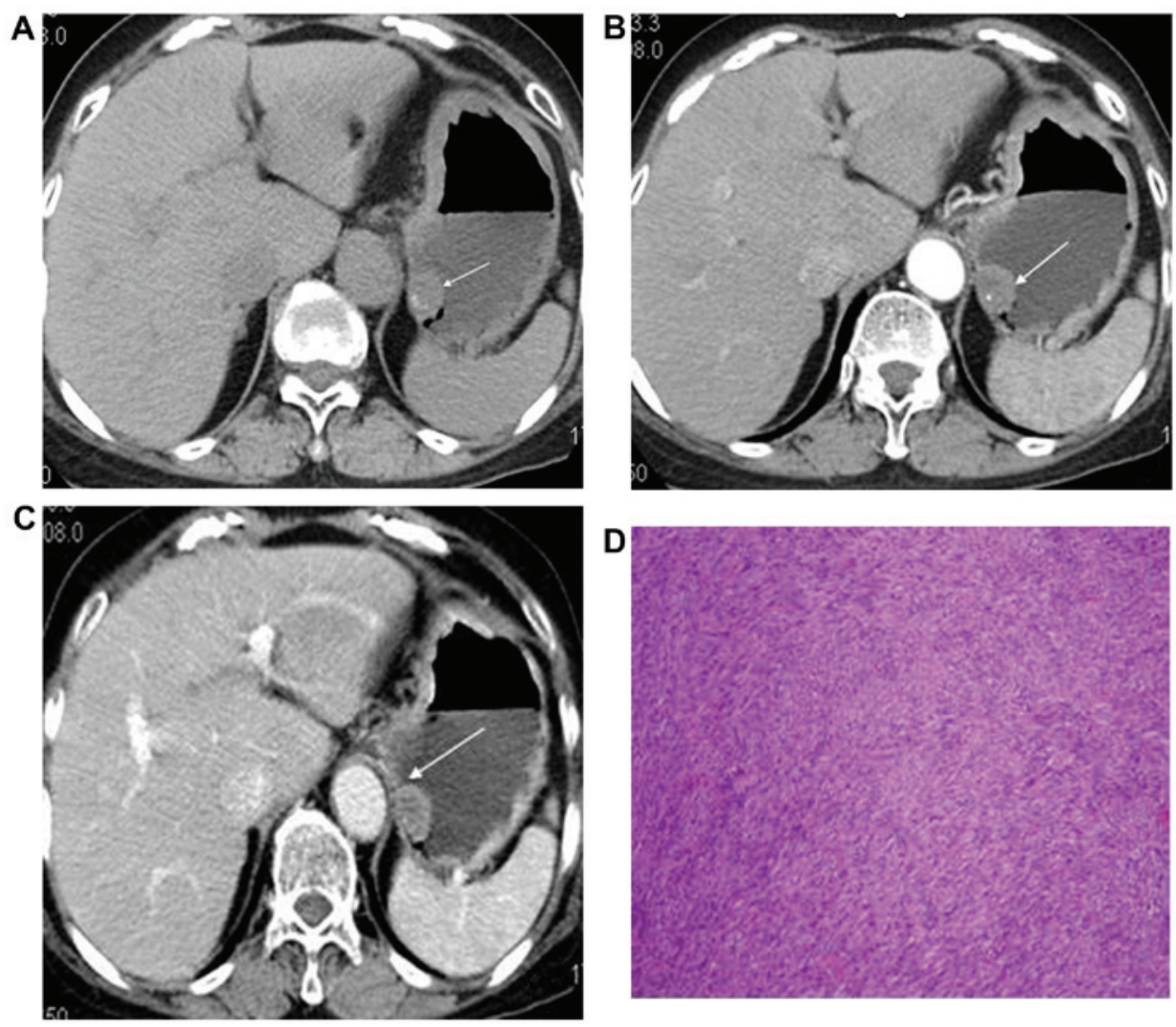

Figure 1. Gastric gastrointestinal stromal tumor (GIST). (A) Computed tomography (CT) of the abdomen in a 51-year-old man complaining of epigastric pain shows an incidental homogeneous ovoid mass with a small punctate calcification in the gastric fundus (arrow). (B and C) Contrast-enhanced CT scan showing slight enhancement of the tumor (arrows). (D) The submucosal nodule was diagnosed as low-risk GIST on histopathological examination. The tumor was composed of cytologically bland spindle cells (hematoxylin and eosin staining; magnification, $\mathrm{x} 100$ ). 


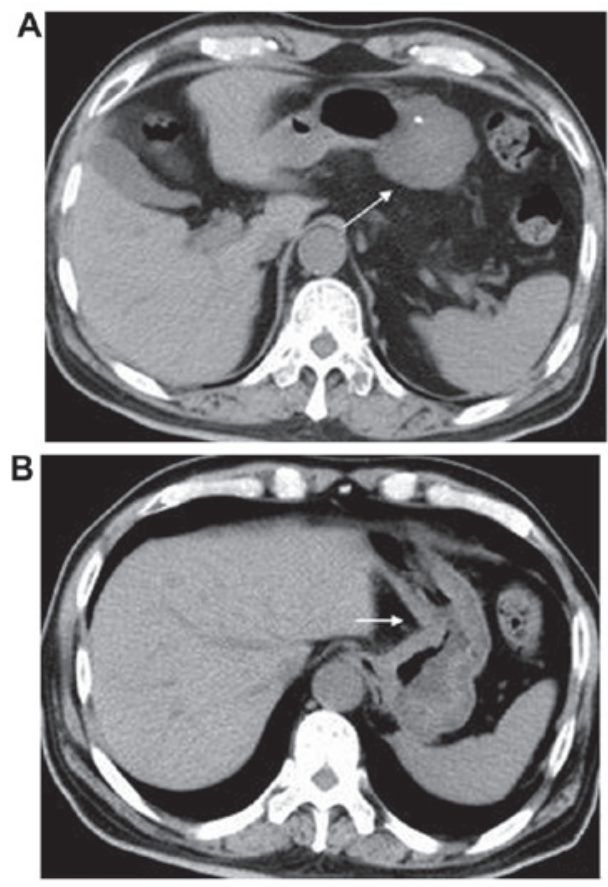

Figure 2. (A) Gastrointestinal stromal tumor arising from the gastric antrum in a 78-year-old man. Computed tomography (CT) scan showing a highly homogeneous mass with punctate calcification (arrow). (B) Gastric cancer. CT scan showing a thickening of the posterior wall of the lesser curvature of the stomach (arrow).

gastroscopy biopsy fragments only showed adenocarcinoma, and most GISTs were detected in the resected stomach. Only in 18 patients $(18 / 26,69 \%)$ were the GISTs identified on preoperative $\mathrm{CT}$.

GISTs $<1.0 \mathrm{~cm}$ could not be detected on preoperative CT, as the tumors are difficult to differentiated from perigastric metastatic LNs and may only confirmed on histopathological examination. The individual CT characteristics of GISTs and regional LN enlargement, which is a significantly more common finding, overlap; therefore, CT cannot reliably differentiate between the two based on the size or pattern of their contour alone. Imaging characteristics that may be associated with LN metastases include multiple lesions, strong enhancement and hypodense areas.

No evidence of necrosis, hemorrhage, or cystic degeneration was found in any of the patients, irrespective of the tumor size $(>5$ or $<5 \mathrm{~cm})$. These CT imaging characteristics were different from those of conventional GISTs (8). The tumor exhibited homogeneous slight or moderate enhancement and it was clinically more common in men compared with women; the median age at the time of diagnosis was 69.2 years in our patients, as previously reported by Fletcher et al (9).

Immunostaining for CD34, c-kit, SMA and S-100 may be diagnostically useful. Rabin et al (1) reported that $40-70 \%$ of GISTs were positive for CD34, 20-30\% were positive for SMA, and $10 \%$ were positive for S-100 protein; In our study, $100 \%$ of the tumors were positive for CD34 and CD117, and 28\% were positive for SMA. The positivity for CD34 and CD117 markers was higher compared with what was previously reported.

The biological behavior of GISTs is often difficult to predict on MSCT. Tumor size and mitotic rate are most frequently used to predict malignant behavior (10). However, as benign-appearing GISTs (small size and absent mitoses or low mitotic rate) may recur or metastasize, a recently published consensus statement suggests classifying GISTs as very low-, low-, intermediate- and high-risk, rather than as benign or malignant (9). All the tumors in our study exhibited $\leq 5$ mitoses/50 hpf and were classified as being of low or very low malignant potential.

In summary, the synchronous occurrence of GISTs and other primary tumors may be more common than what was previously considered and they are usually discovered incidentally during surgery performed for the primary malignancy. Simple coincidence appears to be the most likely explanation, although gene mutations or neighboring gastric tissues affected by the same carcinogen are other hypotheses reported in the literature $(2,11,12)$. A combined genetic deregulation appears to be involved in the pathogenesis of these two entities. Further studies are required to elucidate the molecular and genetic mechanisms underlying carcinogenesis and cancer progression associating GIST and other synchronous tumors.

\section{Acknowledgements}

This study was supported by a grant from the scientific research programs of Fujian provincial Health and Family Planning Commission for young scholars (2014-01-45). We are particularly grateful to Dr Shi Sumeng, for her advice and support at various stages of the project.

\section{References}

1. Rabin I, Chikman B, Lavy R, Sandbank J, Maklakovsky M, Gold-Deutch R, Halpren Z, Wassermann I and Halevy A: Gastrointestinal stromal tumors: A 19 year experience. Isr Med Assoc J 11: 98-102, 2009.

2. Maiorana A, Fante R, Maria Cesinaro A and Adriana Fano R: Synchronous occurrence of epithelial and stromal tumors in the stomach: A report of 6 cases. Arch Pathol Lab Med 124: 682-686, 2000.

3. Rauf F, Ahmad Z, Muzzafar S and Hussaini AS: Synchronous occurrence of gastrointestinal stromal tumor and gastric adenocarcinoma: A case report. J Pak Med Assoc 56: 184-186, 2006.

4. Al-Brahim N, Radhi J and Gately J: Synchronous epithelioid stromal tumour and lipoma in the stomach. Can J Gastroenterol 17: 374-375, 2003.

5. Bircan S, Candir O, Aydin S, Başpinar S, Bülbül M, Kapucuoğlu N, Karahan N and Ciriş M: Synchronous primary adenocarcinoma and gastrointestinal stromal tumor in the stomach: A report of two cases. Turk J Gastroenterol 15: 187-191, 2004.

6. Liu SW, Chen GH and Hsieh PP: Collision tumor of the stomach: a case report of mixed gastrointestinal stromal tumor and adenocarcinoma. J Clin Gastroenterol 35: 332-334, 2002.

7. Yamamoto D, Hamada Y, Tsubota Y, Kawakami K, Yamamoto C and Yamamoto M: Simultaneous development of adenocarcinoma and gastrointestinal stromal tumor (GIST) in the stomach: Case report. World J Surg Oncol 10: 6, 2012.

8. Ulusan S, Koc Z and Kayaselcuk F: Gastrointestinal stromal tumours: CT findings. Br J Radiol 81: 618-623, 2008.

9. Fletcher CD, Berman JJ, Corless C, Gorstein F, Lasota J, Longley BJ, Miettinen M, O'Leary TJ, Remotti H, Rubin BP, et al: Diagnosis of gastrointestinal stromal tumors: A consensus approach. Int J Surg Pathol 10: 81-89, 2002.

10. Miettinen M, El-Rifai W, H L Sobin L and Lasota J: Evaluation of malignancy and prognosis of gastrointestinal stromal tumors: A review. Hum Pathol 33: 478-483, 2002.

11. Lin YL, Tzeng JE, Wei CK and Lin CW: Small gastrointestinal stromal tumor concomitant with early gastric cancer: A case report. World J Gastroenterol 12: 815-817, 2006.

12. Andea AA, Lucas C, Cheng JD and Adsay NV: Synchronous occurrence of epithelial and stromal tumors in the stomach. Arch Pathol Lab Med 125: 318-319, 2001. 